\title{
Diacronie
}

Studi di Storia Contemporanea

$N^{\circ} 34,2$ | 2018

Scuola e società in Italia e Spagna tra Ottocento e Novecento

\section{Alexandra Kollontaï}

Stato comunista e liberazione femminile

\section{Martina Ricci}

\section{(2) OpenEdition}

\section{Journals}

\section{Edizione digitale}

URL: http://journals.openedition.org/diacronie/8609

DOI: $10.4000 /$ diacronie.8609

ISSN: 2038-0925

Editore

Association culturelle Diacronie

\section{Notizia bibliografica digitale}

Martina Ricci, «Alexandra Kollontaï », Diacronie [Online], Nㅜ 34, 2 | 2018, documento 12, Messo online il 29 juin 2018, consultato il 01 mai 2019. URL : http://journals.openedition.org/diacronie/8609; DOI :

10.4000/diacronie.8609 


\title{
Diacronie
}

Studi di Storia Contemporanea

34, $2 / 2018$

Scuola e società in Italia e Spagna tra Ottocento e Novecento

\section{Alexandra Kollontaï: Stato comunista e liberazione femminile}

\author{
Martina RICCI
}

Per citare questo articolo:

RICCI, Martina, «Alexandra Kollontaï: Stato comunista e liberazione femminile», Diacronie. Studi di Storia Contemporanea : Scuola e società in Italia e Spagna tra Ottocento e Novecento, 34, 2/2018, 29/06/2018,

URL: < http://www.studistorici.com/2018/06/29/ricci_numero_34/ >

Diacronie Studi di Storia Contemporanea $\rightarrow$ http://www.diacronie.it

Rivista storica online. Uscita trimestrale.

redazione.diacronie@hotmail.it

Comitato di direzione: Naor Ben-Yehoyada - João Fábio Bertonha - Christopher Denis-Delacour - Maximiliano Fuentes Codera Anders Granås Kjøstvedt - John Paul Newman - Deborah Paci - Niccolò Pianciola - Spyridon Ploumidis - Wilko Graf Von Hardenberg

Comitato di redazione: Jacopo Bassi - Luca Bufarale - Gianluca Canè - Fausto Pietrancosta - Alessandro Salvador - Matteo Tomasoni - Luca Zuccolo

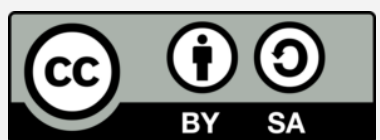

Diritti: gli articoli di Diacronie. Studi di Storia Contemporanea sono pubblicati sotto licenza Creative Commons 3.0. Possono essere riprodotti e modificati a patto di indicare eventuali modifiche dei contenuti, di riconoscere la paternità dell'opera e di condividerla allo stesso modo. La citazione di estratti è comunque sempre autorizzata, nei limiti previsti dalla legge. 


\section{0/ Alexandra Kollontaï: Stato comunista e liberazione femminile}

Martina RICCI

Dopo aver citato alcuni contributi biografici su Tina Anselmi, l'articolo tenta, dapprima, di proporre una sua biografia scandita dai momenti essenziali e, in secondo luogo, di rintracciare le istanze della soggettività dell'Anselmi nella legge sulla parità di trattamento nel Lavoro del 1977, inserendo il tema nel contesto internazionale caratterizzato dal dibattito sulla condizione femminile dopo il 1975, International Women's Year. L'ultima parte, infine, è dedicata al ricordo di una "madre della Repubblica italiana".

\section{Introduzione}

Alexandra Kollontaï è stata uno dei personaggi più importanti della Rivoluzione d'ottobre. Nata nel 1872 in una famiglia di antica nobiltà russa fu una di quelle persone che «usarono coerentemente il privilegio di provenire da una famiglia borghese per formarsi una coscienza critica e che per tutta la vita misero le proprie conoscenze al servizio della lotta per un ordinamento sociale più giusto» ${ }^{1}$. Alexandra fin da bambina ha sempre dimostrato grande sensibilità nei confronti delle classi subalterne; la miseria della servitù della casa paterna e l'amatissima istruttrice politicamente impegnata hanno avuto una grande influenza nella sua formazione ${ }^{2}$. Contro il volere della famiglia sposò giovanissima un ingegnere borghese (Kollontaï), con il quale ebbe anche un figlio. Insoddisfatta della vita coniugale, nel 1896 abbandonò la famiglia per studiare economia a Zurigo, dove s'imbatté in un gruppo di profughi politici russi e diventò progressivamente marxista. Cominciò a viaggiare per l'Europa, e lì conobbe e si legò a importanti esponenti del movimento socialista come Vladimir Lenin e Clara Zetkin. Pochi anni dopo era già una delle principali figure del movimento socialista e comunista dei primi decenni

\footnotetext{
${ }^{1}$ RAETHER, Gabriele, Aleksandra Kollontaj: libertà sessuale e libertà comunista, Pomezia, Erre emme, 1996, p. 11.

${ }^{2}$ Cfr. CAVARERO, Adriana, RESTAINO, Franco, Le filosofie femministe. Due secoli di battaglie teoriche e pratiche, Milano, Bruno Mondadori Editore, 2002, p. 127.
} 
del Novecento, questo le costò il carcere e l'esilio dalla madre patria ${ }^{3}$. Nel 1915 si unì ai bolscevichi e alla causa proletaria, senza però abbandonare la lotta femminista. Al contrario, per Alexandra, «la Révolution d'Octobre signifiait la réalisation de ses espoirs d'un ordre social nouveau pour les femmes» ${ }^{4}$. Le sue idee erano una sorta di combinazione tra marxismo e femminismo, lei credeva che il socialismo marxista fosse la migliore via per raggiungere l'emancipazione femminile 5 . Sosteneva l'idea che la liberazione della donna fosse legata inseparabilmente alla liberazione di uomini e donne dal capitalismo ${ }^{6}$. Lei stessa spiegava che:

in Russia c'era già un movimento piuttosto forte costituito da donne di estrazione borghese, ma la mia ideologia marxista mi faceva vedere con estrema chiarezza che la liberazione della donna si poteva raggiungere solo in quanto risultato di una vittoria che imponesse un nuovo ordine sociale ed un nuovo sistema economico?.

Al momento della presa di potere bolscevica, ad Alexandra fu affidato il Dipartimento degli affari sociali. Fu la prima donna della storia a ricoprire una carica così alta. Essa svolse a pieno il suo compito di commissario redigendo un progetto di decreti che cambiarono radicalmente le condizioni della donna ${ }^{8}$. Alexandra credeva che la lotta femminista dovesse essere combattuta su due fronti: la liberazione economica e l'emancipazione sessuale. Secondo lei, l'indipendenza economica non era sufficiente per risolvere i problemi delle donne. Marxista convinta, si aspettava che una rivoluzione socialista e sessuale avrebbe portato alla distruzione della società di classe e della famiglia'. Solo il tempo avrebbe dimostrato che non era così semplice, che vi erano altre priorità. Lei stessa afferma di essersi resa conto «di quanto poco il partito si preoccupasse del destino delle donne della classe operaia e quanto limitato fosse il suo interesse alla liberazione delle donne» ${ }^{10}$. Alexandra ha espresso instancabilmente le sue idee, e questo spesso l'ha portata contro i suoi stessi compagni, che forse non erano pronti a delle visioni così rivoluzionarie. Negli anni Venti, le questioni sociali ed economiche furono oggetto di controversie all'interno del Partito $^{11}$, e come vedremo, questo avrà delle ripercussioni su Alexandra. Rimase al governo con Lenin fino al 1922, "quando le difficoltà incontrate dalle sue iniziative in favore

\footnotetext{
${ }^{3}$ Cfr. ibidem.

${ }^{4}$ BAILES, Kendall E., IMBERT, Marie-José, «Alexandra Kollontai et la Nouvelle Morale» in, Cahiers du monde russe et soviétique, 6, 4/1965, p. 476.

${ }^{5}$ Cfr. ibidem, pp. 472-473.

${ }^{6}$ Cfr. PORTER, Cathy, Alexandra Kollontai: a biography, London, Virago, 1980, p. 17.

${ }^{7}$ KOLLONTAÏ, Alexandra, Autobiografia di una comunista sessualmente emancipata, Milano, Palazzi, 1973 , p. 42.

${ }^{8} \mathrm{Cfr}$. BAILES, Kendall E., IMBERT, Marie-José, op. cit., pp. 471-472.

${ }^{9}$ STORA-SANDROR, Judith, Alexandra Kollontai: marxisme et révolution sexuelle, Paris, Maspero, 1973, p. 9.

${ }^{10}$ KOLLONTAÏ, Alexandra, op. cit., p. 42.

${ }^{11}$ Cfr. BAILES, Kendall E., IMBERT, Marie-José, op. cit., pp. 471-473.
} 
dell'emancipazione femminile la costringono a dimettersi e ad intraprendere la carriera di ambasciatrice all'estero» ${ }^{12}$.

In questo articolo, verranno illustrate le riflessioni di Alexandra Kollontaï sull'emancipazione femminile e il suo progetto iniziale per la liberazione economica delle donne. Mi soffermerò più precisamente sulle sue elaborazioni riguardanti il lavoro, la famiglia e lo Stato. Ci concentreremo sul suo scritto «La famille et l'Etat communiste». Il periodo storico preso in considerazione dall'articolo corrisponde quindi ai primi anni della giovane repubblica sovietica, dal consolidamento del regime fino alla stalinizzazione. Gli anni che ci interessano di più sono quelli dal 1918 al 1922, periodo di maggiore attività politica di Madame Kollontaï. Il primo paragrafo tratterà del lavoro salariato come strumento di liberazione femminile, il secondo focalizzerà l'attenzione sul lavoro domestico e sull'idea di una nuova struttura familiare e statale, infine verranno affrontate le difficoltà riscontrate da Alexandra e la sua eredità .

\section{Il lavoro: uno strumento di emancipazione e "liberazione"}

Nei decenni a cavallo fra Ottocento e Novecento nei movimenti socialisti era emerso il problema legato all'emancipazione femminile. Gli stessi Marx e Engels avevano indicato «nello stato dei rapporti fra donne e uomini un criterio per misurare e valutare il grado di civiltà di una società, denunciando con lucidità lo stato di degradazione morale e di schiavitù materiale, cui la società capitalistica costringe la maggior parte delle donne» ${ }^{13}$. Secondo loro l'emancipazione della donna poteva essere raggiunta solo attraverso la lotta del proletariato. Engels vedeva l'origine della schiavitù femminile nella proprietà privata, «quindi la rivoluzione socialista e l'abolizione della proprietà privata avrebbero messo fine alla schiavitù della donna, e non solo di quella proletaria $»^{14}$. Nella nascente Repubblica Sovietica la rivoluzione proletaria era già avvenuta, e si doveva quindi pensare a quali percorsi intraprendere per raggiungere la vera liberazione femminile. Alexandra aveva le idee chiare;

ero incline sempre più dell'idea che doveva venire il momento in cui la donna potesse essere giudicata con lo stesso metro morale degli uomini: poiché non é la sua tipica pazienza femminile che le conferisce un posto d'onore nella società degli uomini, bensì l'utilità del lavoro da essa compiuto, il valore della sua personalità intesa come essere umano, come cittadino, come essere pensante, come colui che combatte ${ }^{15}$.

\footnotetext{
${ }^{12}$ CAVARERO, Adriana, RESTAINO, Franco, op. cit., p. 127.

${ }^{13}$ Ibidem, p. 15.

${ }^{14}$ Ibidem, p. 17.

${ }^{15}$ KOLLONTAÏ, Alexandra, op. cit., p. 24.
} 
Il lavoro quindi diventa la chiave. Esso è l'elemento centrale del pensiero comunista, quale fonte di autorealizzazione e di partecipazione alla società ed alla vita comune. Non deve stupirci quindi che diventi anche lo strumento di liberazione principale per le donne.

Al fine di comprendere al meglio il pensiero di Alexandra è importante chiarire la situazione lavorativa femminile in Russia nei primi anni del novecento. In effetti, c'erano stati grandi cambiamenti, a causa della guerra «tra il 1914 e il 1918 il lavoro femminile in Russia era aumentato dal 70 al 400 per cento, a seconda dei settori» ${ }^{16}$. La posizione sociale della donna si era dunque trasformata radicalmente in pochi anni, «già nella Russia prerivoluzionaria le lavoratrici delle industrie tessili rappresentavano il gruppo quantitativamente più forte e più combattivo»" ${ }^{17}$. Anche le donne cominciavano ad essere un attore sociale importante, ci basti pensare che la rivoluzione di febbraio è cominciata proprio da uno sciopero di operaie. Con l'instaurazione del potere rivoluzionario vi è stata l'introduzione dell'«obbligo generale al lavoro» e questo fu una svolta decisiva, secondo l'ottica della nostra autrice, in direzione dell'emancipazione della donna $^{18}$. Alexandra vede nel lavoro il principale ruolo liberante per le donne siccome dona possibilità di creatività e assunzione di responsabilità ${ }^{19}$. Nel nuovo modello di società proposto da Madame Kollontaï: «la femme de la cité communiste ne dépend plus de son mari, mais de son travail, ce n'est pas son homme, ce sont ses bras d'ouvrière qui la nourrissent» ${ }^{20}$.

La "nuova donna" socialista proposta da Madame Kollontaï è quindi un soggetto autosufficiente e autonomo della società. Lei crede che il lavoro sia lo scopo principale dell'esistenza $^{21}$. Esso non rappresenta solo una conquista d'indipendenza economica, ma assume anche il ruolo di strumento d'autorealizzazione personale e di partecipazione alla vita politica. Infatti nel 1918 Alexandra fu tra le organizzatrici del Primo Congresso delle donne lavoratrici russe dal quale nacque lo Żenotdel, organismo per la promozione della partecipazione delle donne alla vita pubblica, per le iniziative sociali e la lotta all'analfabetismo ${ }^{22}$. Quest'organo mirava «alla cooperazione politica delle donne in tutte le istituzioni dei soviet» ${ }^{23}$. Negli anni successivi lo Żenotdel organizzava settimanalmente incontri tra delegate, ai quali partecipava una delegata per ogni fabbrica, e bisogna sottolineare il fatto che «la sezione femminile aveva notevole influsso

\footnotetext{
${ }^{16}$ RAETHER, Gabriele, op. cit., p. 35.

${ }^{17}$ Ibidem.

${ }^{18}$ Cfr. ibidem, pp. 40-41.

${ }^{19} \mathrm{Cfr}$. KOLLONTAII, Alexandra, op. cit., pp. 10-12.

${ }^{20}$ Apparso per la prima volta in Le Phare (ètudes et documentation socialistes), 8, $1^{\circ}$ aprile 1920 e in Bulletin communiste, 7, 29 aprile 1920. Il testo però data 1918 in quanto è stato presentato per la prima volta al congresso panrusso degli operaie e delle contadine (16-21 novembre 1918).

${ }^{21}$ Cfr. KOLLONTAÏ, Alexandra, op. cit., pp. 31-32.

${ }^{22}$ Cfr. RAETHER, Gabriele, op. cit., pp. 43-46.

${ }^{23}$ Cfr. ibidem, p. 46.
} 
sulla legislazione sociale e sul diritto di famiglia, come si dimostrò, per esempio, con la legalizzazione dell'aborto (1920) $»^{24}$.

Il lavoro quindi, era fonte di emancipazione domestica, partecipazione alla vita politica e di autorealizzazione personale. Ciononostante bisogna dire che la possibilità di scegliere un'“attività amata" sarebbe rimasta un privilegio di pochi. Inoltre, perché il lavoro possa realmente essere uno strumento di liberazione bisognava risolvere, secondo la Kollontaï, il problema del "lavoro domestico". Come si può pensare ad una vera partecipazione delle donne alla società comunista visto l'alto numero di ore occupato del lavoro domestico? Il carico di lavoro domestico che gravava sulle donne lavoratrici era un problema che il nuovo Stato comunista avrebbe dovuto risolvere secondo Alexandra.

\section{Stato, famiglia e lavoro domestico}

Come abbiamo già rilevato le questioni sociali, e soprattutto il tema della famiglia e del matrimonio, erano i prediletti di Alexandra. Ora focalizzeremo l'attenzione sulla sua elaborazione inerente al lavoro domestico, alla famiglia e allo stato a partire dal testo del 1918 La Famille et l'Etat communiste. Secondo Madame Kollontaï la forma tradizionale della famiglia si distrugge progressivamente in tutti i paesi nei quali regna il capitale. Afferma che "plus le travail salarié de la femme se généralise et plus aussi la famille se décompose $\aleph^{25}$. Prima dell'avvento del capitalismo, solo l'uomo era il sostegno familiare (nel senso di apporto di capitale). Con le difficoltà economiche che emergono dall'instaurazione dei regimi capitalisti, Kollontaï spiega che siccome il solo salario dell'uomo non è più sufficiente a provvedere ai bisogni familiari cosicché anche la donna è obbligata a cercare un lavoro remunerato fuori di casa ${ }^{26}$.

La donna, investendo tutte le ore a disposizione nel lavoro salariato, smette quindi di lavorare per il nucleo domestico. La donna prima produceva marchandise per l'economia domestica ${ }^{27}$, come ad esempio candele, alimenti in conserva, filo da tessitura etc. La merce in eccesso era venduta in piccoli mercati. Secondo lei lo stato beneficiava del lavoro non remunerato delle donne, poiché non doveva provvedere a questi bisogni della popolazione che venivano soddisfatti grazie al lavoro domestico delle donne ${ }^{28}$. Come mai questo cambio di paradigma? Il capitalismo ha sostituito questo lavoro con le fabbriche, «tout ce qui auparavant se faisait au sein de la famille a été fabriqué en masse, dans les ateliers et fabriques» ${ }^{29}$. Ora sono le operaie e gli operai nelle

\footnotetext{
${ }^{24}$ Ibidem.

${ }^{25}$ KOLLONTAÏ, Alexandra, op. cit., p. 2.

${ }^{26} \mathrm{Cfr}$. ibidem.

${ }^{27}$ Cfr. ibidem, p. 3.

${ }^{28} \mathrm{Cfr}$. ibidem, p. 3.

${ }^{29}$ Ibidem.
} 
fabbriche che producono questo genere di beni. C'è quindi un cambiamento importante: «la famille consomme, mais elle ne produit plus» ${ }^{30}$.

Le conseguenze di questo cambiamento di paradigma per le donne sono state importanti, esse si sono ritrovate a dover svolgere più compiti allo stesso momento. Secondo Madame Kollontaï il lavoro domestico non è più produttivo, e la donna si ritrova a dover compiere tre compiti alla volta: fornire delle ore di lavoro, il menage domestico, e prendersi cura dei figli ${ }^{31}$. Il capitalismo ha quindi messo un grande fardello sulle spalle delle donne, le ha rese delle salariate, pur restando schiave del carico domestico.

Per Madame Kollontaï la soluzione sembra essere la costruzione di una società comunista. Infatti, secondo lei, in una società comunista la lavoratrice non dovrà più sprecare le sue ore riservate allo svago a cucinare siccome ci saranno dei ristoranti pubblici e delle cucine centrali dove tutti potranno andare a prendere i pasti ${ }^{32}$. Nella società comunista di domani la donna non dovrà occuparsi nemmeno dell'abitazione, poiché ci sarà una categoria di lavoratori specializzati che si occuperà anche di quello. Ciò che lei prevede sono quindi dei "servizi collettivi" che si sostituiranno ai lavori domestici a carico delle donne, quindi una trasformazione delle attività domestiche in un'economia socializzata, con la creazione di: cucine comuni e refettori pubblici, di lavanderie, sartorie, asili e nidi d'infanzia, collegi e istituti scolastici di vario tipo ${ }^{33}$. La forza lavoro della donna doveva essere, come quella di ogni altro individuo, completamente a disposizione dello Stato socialista e «per incrementare il loro rendimento professionale si doveva garantire alle donne libero accesso all'istruzione» ${ }^{34}$. La Kollontaj «vedeva il progresso esistenziale per la donna, da un lato, nella possibilità di svolgere, al di là dell'ambito privato familiare, un lavoro utile e socialmente riconosciuto; dall'altro, nella conquista dell'indipendenza economica dall'uomo» ${ }^{35}$. Precisava inoltre che «l'uguaglianza economica e politica della donna poteva comunque realizzarsi soltanto se la maternità veniva considerata una funzione sociale e in quanto tale, protetta dallo Stato» ${ }^{36}$.

Ricapitolando, Alexandra prevedeva la fine della struttura familiare esistita fino a quel momento: «déjà ses bases avaient été sapées par le nombre croissant des femmes salariées, et dans la nouvelle société les assises matérielles de la famille disparaitraient complètement» ${ }^{37}$. Inoltre, il vecchio sistema dei lavori domestici sarà rimpiazzato dai servizi collettivi di lavanderia, cucina, per la pulizia domestica. Tutto il lavoro del menage domestico, cura ed educazione dei figli

\footnotetext{
${ }^{30}$ Ibidem.

${ }^{31}$ Cfr. ibidem, p. 2.

${ }^{32}$ Cfr. ibidem, pp. 3-4.

${ }^{33}$ Cfr. BAILES, Kendall E., IMBERT, Marie-José, op. cit., p. 476.

${ }^{34}$ RAETHER, Gabriele, op. cit., p. 40.

${ }^{35}$ Ibidem, p. 41.

${ }^{36}$ Ibidem.

${ }^{37}$ BAILES, Kendall E., IMBERT, Marie-José, op. cit., p. 476.
} 
compresi, saranno assicurati dalla società ${ }^{38}$. Questo processo avrebbe assicurato alle donne maggior tempo a disposizione, e maggior libertà individuale per diventare un individuo realizzato e partecipativo nella società. Il tempo ci ha dimostrato che il pensiero di Alexandra era forse troppo ottimista.

\section{Tra realtà e utopia}

Madame Kollontaï riuscì in un primo momento a varare delle riforme radicali, quali la legge sul matrimonio, il divorzio, e l'aiuto statale alle madri.

I primi anni del regime sovietico hanno realmente sconvolto strutture millenarie di subordinazione schiavistica delle donne in quel paese consentendo loro di accedere non solo al voto, ma anche alle professioni, alle cariche istituzionali, e di liberarle, in parte, dai pesi della famiglia con la costruzione di servizi sociali molto diffusi soprattutto nelle città39.

Nel periodo post-rivoluzione stava emergendo una nuova società basata sulla valorizzazione dell'individuo tramite il lavoro, ed il raggiungimento dell'emancipazione femminile sembrava fosse all'orizzonte. Alexandra, infatti, scrisse «La famille et l'Etat comuniste» nel 1918, quando ancora vi erano grandi presupposti e grandi speranze per la Rivoluzione Bolscevica. Con il tempo, lei stessa si rese conto che l'emancipazione femminile non si sarebbe acquisita così facilmente. Nonostante i cambiamenti radicali, soprattutto per quanto riguardava lo statuto giuridico, «le donne continuarono a sbrigare i quattro quinti del lavoro domestico: evidentemente disponevano meno tempo degli uomini per attività politiche e sociali in genere», e «nella coscienza delle persone e nel rapporto tra i sessi è rimasto quasi tutto come prima $»^{40}$. Or dunque, «l'espropriazione degli espropriatori (Marx) non porta automaticamente alla liberazione della donna» ${ }^{41}$.

Alexandra rendendosi conto di queste difficoltà nel 1926 scriveva:

Ora, se è vero che il lavoro è senz'altro uno strumento di emancipazione, in quanto, permettendo alle donne l'indipendenza economica, le emancipa dalla schiavitù domestica e dall'asservimento emotivo nel matrimonio, è anche vero che, nei confronti di un rapporto amoroso liberamente scelto e non istituzionalizzato, è solo parzialmente un mezzo di liberazione, perché non è sufficiente, da solo, a determinare la presa di coscienza dei modelli

\footnotetext{
${ }^{38}$ Cfr. ibidem, p. 476.

${ }^{39}$ CAVARERO, Adriana, RESTAINO, Franco, op. cit., p. 19.

${ }^{40}$ RAETHER, Gabriele, op. cit., pp. 104-105.

${ }^{41}$ Ibidem, p. 105.
} 
discriminatori che sopravvivono all'interno della coppia eterosessuale. I limiti dell'attività extra-domestica, come strumento di liberazione, appaiono evidenti nei paesi socialisti europei, dove la condizione delle donne è indubbiamente paritaria per quanto riguarda le possibilità di lavoro e la legislazione (pieno impiego, diritto di aborto, divorzio), ma è rimasta ancorata ai modelli tradizionali per quanto riguarda il loro ruolo all'interno della struttura famigliare ${ }^{42}$.

Madame Kollontaï si rese conto che la lotta per la "liberazione" femminile doveva ancora essere combattuta, e soprattutto avrebbe richiesto una trasformazione delle strutture più radicate della società. Non bastava un cambiamento economico e politico, non bastavano le riforme sul codice civile. Ci voleva un mutamento della mentalità. Mancava inoltre il sostegno da parte del potere politico. Seppur inizialmente la causa femminile ebbe il sostegno di Lenin, con la necessità di consolidamento del potere bolscevico emersero altre priorità. La nascente Unione Sovietica non aveva le possibilità di attuare materialmente tutte le riforme pensate da Alexandra, quindi la lotta femminista e la questione della liberazione della donna rimasero temi secondari. Cambiare realmente la mentalità ed i rapporti sociali non è mai stata una possibilità reale.

La Kollontaï cominciò a scontrarsi con il partito non solo sulla questione femminile, ma anche per la politica del partito bolscevico. Nel 1921 si dimise dalla carica di commissaria del popolo in segno di protesta alla firma del trattato di Brest-Litovsk. Nello stesso anno guidava con Alexandre Šljapnikov Opposizione Operaia; una frazione del partito che si opponeva alla nuova politica economica, rivendicava il controllo dell'industria da parte dei sindacati un maggiore controllo dei lavoratori del partito ${ }^{43}$. Alexandra aveva un'immensa fiducia nelle capacità creatrici delle masse e una grande diffidenza nei confronti delle forme burocratiche ${ }^{44}$. Dopo aver partecipato al movimento di Opposizione Operaia Alexandra fu allontanata e si allontanò sempre più dal partito. Madame Kollontaï cominciò a concentrarsi sulle questioni femminili, lasciando a distanza il partito ed il socialismo. Iniziò a scrivere testi e romanzi sulla questione della liberazione femminile, la sessualità e la nuova morale che avrebbe dovuto instaurarsi nella società sovietica.

\section{Eredità}

La questione della ricezione del pensiero di Alexandra Kollontaï è un punto critico. Forse in quanto donna spesso è stata trattata in maniera diversa, sicuramente non sempre le sue riflessioni hanno avuto le considerazioni che meritavano e probabilmente è anche per questo che è uscita

\footnotetext{
${ }^{42}$ KOLLONTAÏ, Alexandra, op. cit., p. 11.

${ }^{43}$ Cfr. BAILES, Kendall E., IMBERT, Marie-José, op. cit., p. 472.

${ }^{44}$ Ibidem, p. 477.
} 
così modestamente dalle purghe di Stalin. Purtroppo la relazione tra femminismo e socialismo è spesso stata conflittuale, e l'Unione Sovietica non sfugge certo a questa difficoltà. A partire dal 1925 Madame Kollontaï iniziò a lavorare come ambasciatrice per l'Unione Sovietica, e fu così che riuscì a raggiungere un nuovo obbiettivo: fu la prima donna a ricoprire quel ruolo. Non smise mai di lottare per la causa femminista, e continuo a pubblicare romanzi e articoli nel corso degli anni.

Alexandra è stata un personaggio importante per l'emancipazione femminile, non solo per le donne delle Repubbliche Sovietiche, ma anche per quelle degli stati europei occidentali. Le sue proposte di legge sono state riprese da questi ultimi con il sistema del Welfare State. Eppure oggi sembra che pochi conoscano il suo nome. Nonostante i traguardi raggiunti, «la speranza di Alexandra Kollontaï di essere accolta nel novero di coloro che verranno recepiti e studiati dalle generazioni successive come "oggetti di un importante periodo storico", come immagine e modello, finora non si è realmente verificata $»^{45}$. Madame Kollontaï e la sua opera hanno avuto un breve periodo di gloria all'inizio degli anni settanta, ma sono oggi quasi completamente cadute nell'oblio ${ }^{46}$. La causa è da ricercare nel "divorzio" tra femminismo e socialismo avvenuto in quegli anni. Nel corso degli anni settanta c'è stato un momento di crescente disinteresse per le teorie socialiste. I femminismi prima erano spesso vicini a correnti di sinistra e lottavano attraverso l'economia politica con l'obiettivo di cambiare la società dalla radice ${ }^{47}$. Tuttavia «quando le energie utopiste iniziarono a declinare il femminismo di seconda ondata fu inglobato nell'orbita della politica identitaria» ${ }^{48}$. È venuta a crearsi una collaborazione ambivalente tra i movimenti emancipatori degli anni settanta ed il paradigma neoliberale. Le femministe di questi anni, di fronte all'impossibilità di eliminare le strutture di dominio nel sistema economico, hanno abbandonato la lotta al capitale per concentrarsi sulla riflessione identitaria e sulla lotta per il riconoscimento ${ }^{49}$.

Alexandra di conseguenza non è entrata nelle antologie femministe, o perlomeno solo in quelle più vicine alla sinistra. Rimane comunque una donna cha ha cambiato radicalmente la condizione femminile in Unione sovietica e non solo. È importante quindi ricordare tutte le battaglie combattute, le sconfitte subite e le vittorie guadagnate. L'esperienza di Alexandra ci dimostra che il cambiamento delle strutture materiali non basta per raggiungere l'uguaglianza, ci voleva anche una lotta più profonda e mirata alla distruzione delle strutture mentali che promuovono la discriminazione femminile.

\footnotetext{
${ }^{45}$ RAETHER, Gabriele, op. cit., p. 7.

${ }^{46} \mathrm{Cfr}$. ibidem.

${ }^{47}$ Cfr. FRASER, Nancy, «Oltre l'ambivalenza: la nuova sfida del femminismo», in Scienza \& Politica, XXVIII, 54, 2016, pp. 90-92.

${ }^{48}$ Ibidem, p. 90.

${ }^{49}$ Cfr. ibidem, pp. 92-96.
} 


\section{Conclusioni}

Il personaggio di Alexandra Kollontaï è sicuramente uno dei più interessanti ed emozionanti della Rivoluzione russa. Nonostante spesso venga dimenticata, è stata una grande militante per l'instaurazione del potere socialista, un'ottima oratrice con la capacità di ammaliare le masse e una partigiana della lotta per l'emancipazione femminile. Alexandra ha lottato instancabilmente anche contro i suoi stessi compagni per difendere le sue tesi e la sua ricerca della libertà politica e sessuale.

Il testo da noi analizzato, «La Famille et l'Etat communiste», ci mostra Alexandra in gioventù, con ancora grandi speranze ed ottimismo. Già nell'autobiografia del 1926 Alexandra ha perso quell'illusione giovanile ed il tono è molto più realistico e meno entusiastico. Nelle sue riflessioni il lavoro era fonte di emancipazione domestica, partecipazione alla vita politica e di autorealizzazione personale. Se esso fosse stato accompagnato dalle adeguate politiche statali forse l'emancipazione femminile sarebbe stata raggiunta. La realtà pose subito Alexandra di fronte alle difficoltà materiali e sociali delle sue iniziative. In effetti, troviamo un'Unione Sovietica che non ha le potenzialità materiali per soddisfare i bisogni delle nuove strutture statali pensate da Alexandra. Nonostante dei cambiamenti radicali fossero stati introdotti, come ad esempio le leggi sul divorzio, il matrimonio, il supporto alla maternità, etc, la mentalità ed i comportamenti paternalistici non sono scomparsi in Unione sovietica, né nella vita privata e tantomeno in quella pubblica. In realtà, seppur le donne in Unione sovietica godessero di uno statuto paritario di tutto rispetto, se confrontato agli altri paesi in quel periodo, le donne sovietiche non hanno mai goduto della vera parità di trattamento. E questo dimostra che non basta cambiare le strutture economiche e sociali per avere un cambiamento di mentalità immediato. D'altra parte dimostra anche che vi erano altre priorità a cui far fronte secondo l'ordine del giorno del partito. Le questioni sociali non hanno mai beneficiato di priorità, soprattutto dopo l'instaurazione del potere staliniano e i piani quinquennali, quando addirittura si tornò alla criminalizzazione dell'aborto e all'indurimento dei compiti matrimoniali delle donne. La società sovietica forse, e soprattutto il partito comunista, non erano pronti alle riflessioni radicali di Alexandra.

Nonostante tutto, Alexandra ha portato dei miglioramenti nelle condizioni di vita delle donne in Unione sovietica, e potrebbe essere un personaggio di spunto per femministe di ogni epoca. Alexandra anche dopo l'allontanamento dalla Repubblica Sovietica ha continuato ad interessarsi alla lotta per la libertà femminile, ampliando le sue riflessioni (che in questa piccola ricerca non sono state trattate) e fornendo un grande punto di partenza per le future femministe. Il suo nome è quasi sconosciuto alla nostra generazione; probabilmente perché non è diventata martire della rivoluzione come Rosa Luxemburg e anche perché al momento del successo di Alexandra c'è stato un distacco e un rifiuto delle femministe dalle correnti marxiste-socialiste. Di conseguenza 
Madame Kollontaï non ha avuto i riconoscimenti che merita, ma ci lascia comunque con grandi insegnamenti. È importante volgere uno sguardo al passato se l'idea è di costruire il futuro. Ancora siamo lontane dall'equità, non sono state eliminate le cause strutturali della discriminazione. «Il doppio sfruttamento della donna ad opera del capitale e del lavoro non è stato eliminato ${ }^{50}$. Utilizziamo questi spunti di riflessione formulati da donne che hanno instancabilmente combattuto per la liberazione femminile. L'esperienza di Alexandra dimostra che il cambiamento delle strutture materiali non basta per raggiungere l'uguaglianza. La storia a noi più vicina invece ci mostra quanto la lotta giocata solo sul riconoscimento non sia sufficiente. La soluzione sembra essere quella di unire le forze e lottare su più fronti per ottenere sia un cambiamento del sistema economico sia un radicale cambiamento di mentalità.

${ }^{50}$ RAETHER, Gabriele, op. cit., p. 105. 


\section{L'AUTORE}

Martina RICCI è attualmente studentessa del corso di Laurea Magistrale in Scienze Storiche presso l'Università di Bologna, con indirizzo storia contemporanea. Laureata in Relazioni Internazionali presso l'Università di Ginevra s'interessa particolarmente di teoria femminista e di storia delle donne. Ha conseguito una tesi sui femminismi turchi dal titolo: Femmes, Etat e société dans la Turquie contemporaine.

URL: < http://www.studistorici.com/progett/autori/\#Ricci > 